\title{
Persistent Hiccups after Cervical Selective Nerve Root Block
}

\author{
Na Eun Kim, Gyoung A Heo, Byung Gun Kim, Ki Hyun Park, Jae Woung Uhm \\ Department of Anesthesiology and Pain Medicine, Inha University Hospital, Inha University School of Medicine, Incheon, Korea
}

\begin{abstract}
Persistent hiccups are rare complications following epidural steroid injections. Although the underlying etiology is not clearly understood, corticosteroids are the drug group referenced most frequently in the literature as being associated with hiccups. A 54-year-old man occurred a persistent hiccup after cervical root block due to cervical radiculopathy. A stellate ganglion block was performed, but the hiccup continued. After that, the hiccup did not stop, so metoclopramide $10 \mathrm{mg}$ was prescribed. After taking the drug the next day, hiccups started to decrease in frequency, the hiccup was completely stopped from the second day of taking the drug, the hiccup was completely stopped. He reported that he had not experienced recurrent hiccups. This report highlights the importance of evaluating the cause of hiccups and determining the treatment strategy accordingly.
\end{abstract}

Keywords: Hiccup; Cervical nerve root block; Persistent hiccups; Dexamethasone; Metoclopramide

\section{INTRODUCTION}

Hiccups are involuntary contractions of the diaphragm, which is the muscle that separates the chest from the abdomen and plays an important role in breathing. Hiccups are a usually self-limiting disorder that stops within a few minutes to a few hours. In most cases, it is a benign condition that causes no harm to the patient. Hiccups can be classified according to their duration: persistent hiccups that last for more than 48 hours, and intractable hiccups that last for more than 2 months, and they are associated with sleep disturbance, fatigue, and kidney failure [1].

The etiology and mechanism of hiccups are not clearly understood, and over 100 causes have been identified [2,3]. Corticosteroids are the drug group referenced most frequently in the literature as being associated with hiccups. It has been proposed that corticosteroids lower the threshold for synaptic transmission in the midbrain and directly stimulate the hiccup reflex arc [4].

We describe a patient with persistent hiccups as a complication of cervical selective nerve root block and discuss treatment strategies for persistent hiccups.

\section{CASE REPORT}

A 54-year-old man with a past medical history of hypertension presented to the pain clinic for treatment of right arm numbness which continued for approximately 1 month. Analgesics, including nonsteroidal anti-inflammatory drugs and tramadol, did not provide any relief and he did not respond to physiotherapy. Magnetic resonance imaging of his cervical spine showed grade 1 central stenosis at C5-C6 and bilateral grade 2 foraminal stenosis at C6-C7.

First, it was decided to perform a cervical epidural block and was performed at the C7-T1 level using a 16G epidural Tuohy needle under fluoroscopic guidance, and $8 \mathrm{~mL}$ of $0.5 \%$ mepivacaine, $2 \mathrm{mg}$ of dexamethasone, and 1,500 IU of hyaluronidase were administered. The procedure went well without complications. On the next visit, despite the previous treatment, he complained of tingling sense and radicular pain in his arm and neck. So, a cervical root block was performed. A cervical selective nerve root block was performed at the C6 level under fluoroscopic guidance in the supine position, using a 22G epidural Tuohy needle, and $6 \mathrm{~mL}$ total volume of a mixture of $1 \%$ mepivacaine, $5 \mathrm{mg}$ of dexametha- 
sone, and 1,500 IU of hyaluronidase were administered. The needle is advanced under image guidance at an angle of $45^{\circ}$ on the right side until the tip of the needle is just anterolateral to the posterior border of neural foramen, and an anteroposterior view is also obtained at this stage. The needle was advanced to enter the nerve root canal under image guidance, observing the patient's response. The procedure proceeded without bleeding or no neurological complication and the vital signs were stable. The radicular pain to the arm and neck was relieved following the procedure for 2 hours and the patient returned home.

The next day, the patient visited our pain clinic complaining of the hiccups, which were severe and not accompanied by any other symptoms. He said that hiccups started 6 hours after the procedure and lasted until the next day. He complained that he was tired from staying up all night with hiccups. Considering the occurrence of hiccups due to sympathetic nerve irritation during the procedure and stellate ganglion block (SGB) was performed to relieve hiccups using $4 \mathrm{~mL}$ total volume of a mixture of $1 \%$ mepivacaine and $1 \mathrm{mg}$ of dexamethasone.

Transverse process of cervical vertebra (Chassaignac's tubercle) at C6 was felt through deep palpation by pulling a sternocleidomastoid muscle and carotid sheath in the immediately lateral of cricoid and was punctured with a $21 \mathrm{G}$ syringe containing medication, and then advanced until C6 transverse process is contacted. At this point, the needle tip is retracted $1-2 \mathrm{~mm}$ and it is assured blood does not come with aspiration. SGB was performed without complication and the patient's vital signs were stable after the procedure. Nevertheless, hiccups resumed after an hour. The patient was prescribed $10 \mathrm{mg}$ of metoclopramide for every 12 hours for continuous hiccup treatment. The patient's symptoms were relieved after taking the drug and no hiccups occurred again.

The present case report was published with the written informed consent of the patient's guardians.

\section{DISCUSSION}

Hiccups, or singultus, are generally benign, transient events experienced by the normal population and can be caused by various factors such as diet, disease, and stress. Nevertheless, it is unusually idiopathic. Known causes include cancer-related, drug-induced, procedure-induced nerve irritation [4,5]. Most hiccups are thought to be caused by gastrointestinal problems, due to rapid intake of food and increased air inflow. Other causes include gastrointesti- nal infarction or phrenic nerve damage due to cancer, brain tumors, and kidney failure [6].

Three components are involved in the hiccup reflex: the afferent, central, and efferent limbs. Relating to the afferent limb is the phrenic and vagus nerves and the sympathetic chain from the thoracic segments T6-T12. Relating to the efferent limb is the phrenic nerve and its interaction with the glottis, accessory respiratory muscles, and the central processor such as the brainstem, midbrain areas, including the respiratory center, phrenic nerve nuclei, medullary reticular formation, and hypothalamus [4]. However, the mechanisms related to the central processor is still unknown. A hiccup can occur if the above elements are stimulated by various factors.

Dexamethasone has been used as a powerful antiemetic drug during chemotherapy. One study reported that the incidence of hiccups in patients treated with dexamethasone was $42 \%$ [7]. Dexamethasone is a common cause of drug-induced hiccups, and the exact mechanism of action is not known. However, it is known that corticosteroids stimulate the hiccup reflex arch and decrease the threshold of synaptic transmission in the midbrain. In addition, dexamethasone has a unique structure and affinity to the hiccup reflex receptor in the brain stem, compared to other steroid drugs [7].

The administration of epidural steroid injections (ESIs) has increased with the recent increase in the elderly population, and various side effects have been reported, such as weight gain, mild fever, insomnia, and hiccups $[7,8]$. In the case of hiccups caused by ESI as described above, it is necessary to consider both the steroid agent in the mixture and nerve irritation or hyperactivity of the sympathetic nerve when administering a cervical or thoracic ESIs. Slipman et al. [3] first reported that a persistent hiccup occurred when the thoracic epidural block was performed with betamethasone and 1\% lidocaine and persistent hiccups occurred 15-18 hours after the injection. Since the hiccup occurred 16 hours after the thoracic epidural block and the hiccup was also generated in the second same procedure, the steroid agent mixture was the cause of the hiccup [2].

Particulate corticosteroids are considered to be at high risk of ischemic neurologic injury, Non-particulate preparations, such as dexamethasone, are more commonly used in epidural blocks than other steroids. It is possible to increase the occurrence of a druginduced hiccup.

In this case, the cervical epidural steroid mixture may be con- 
Kim NE, et al. - Persistent Hiccups after Cervical Selective Nerve Root Block

sidered the cause of the hiccup, but it is known that the incidence rate increases when a high dose of $10 \mathrm{mg}$ or more is used. The occurrence of a procedure-related hiccup should also be considered.

In the epidural block related hiccup cases, it is explained as the change of volume to the epidural space and altering the balance cerebrospinal fluid volume $[2,8]$. However, in this case, since there were not many volumes injected into the epidural, so it is thought that physical and chemical irritation in the hiccup reflex arch may have been the cause of the hiccup.

In this case, persistent hiccups occurred after the cervical root block. When performing the cervical root block, the needle was inserted to the right at a $45^{\circ}$ angle to stimulate the vagus nerve or phrenic nerve. The phrenic nerve originates from C3-5 ventral ramus travels along the upper lateral border of the anterior scalene muscle and then approaches the medial further along the anterior surface toward the medial border. The pathway of the phrenic nerve may be a stimulus when handling a needle when performing a lower cervical root block. In addition, the drug delivered through the cervical root block may stimulate the anterior part of the spinal root of C3-5, an afferent pathway of the hiccup reflex $\operatorname{arc}[9]$.

However, when the patient re-visited the hospital due to the hiccups, hiccups recurred after SGB with steroid mixture. According to a previous study, blockade of the sympathetic nerves or central limbs in the hiccup arch was shown to alleviate symptoms $[10,11]$. However, the hiccup was continuously generated even after SGB, it can be thought of as either insufficient sympathetic block or triggered again by the used dexamethasone.

Although the exact cause of hiccup has not yet been identified, first of all, based on several cases and studies, the medication should be given, and if hiccup continues to occur afterward, continuous epidural block or SGB can be considered. The treatment for persistent hiccups is shown [11] (Table 1).

In most cases of steroid-induced hiccups, substitution with other types of steroids or combined drug treatment is recommended.

Baclofen was first reported to treat persistent hiccups in nine patients with Launois, and intractable hiccups were also reported to be treated with oral drugs only due to gastroesophageal reflux $[11,12]$. Baclofen is a GABA (gamma-aminobutyric acid) agonist that reduces the excitability of the hiccup reflex and exhibits antispastic efficacy by reducing synaptic transmission to the spinal cord. In addition, anticonvulsants recommended as second-line treatments should be used with caution if the patient exhibits neu-
Table 1. The treatment of persistent hiccups

\begin{tabular}{|c|c|c|c|}
\hline Pharmacological therapy & Dose & $\begin{array}{l}\text { Level of } \\
\text { evidence }\end{array}$ & Concerns \\
\hline \multicolumn{4}{|l|}{ 1st line } \\
\hline Baclofen & $3 \times 5-20 \mathrm{mg} /$ day & $2 b$ & Sedation \\
\hline Gabapentin & $3 \times 300-600 \mathrm{mg} / \mathrm{day}$ & 4 & Sedation \\
\hline Pregabalin & $2 \times 75-150 \mathrm{mg} /$ day & 5 & Sedation \\
\hline \multicolumn{4}{|l|}{ 2nd line } \\
\hline Metoclopramide & $3 \times 10 \mathrm{mg}$ & $2 b$ & \\
\hline Domperidone & $3 \times 10 \mathrm{mg}$ & 5 & Long Q-T syndrome \\
\hline \multicolumn{4}{|l|}{ 3rd line } \\
\hline Chlorpromazine & $4 \times 25-50 \mathrm{mg}$ & 4 & Postual hypotension \\
\hline \multicolumn{4}{|l|}{ Other choice } \\
\hline Amitriptylline & $1 \times 25-100 \mathrm{mg} / \mathrm{night}$ & 5 & Sedation, dry mouth \\
\hline Nifedipine & 60-180 mg/day & 4 & Hypotension \\
\hline \multicolumn{4}{|c|}{ Nonpharmacological therapy } \\
\hline $\begin{array}{l}\text { Nerve block (C3-C5), } \\
\text { phrenic nerve, vagal } \\
\text { nerve }\end{array}$ & & 5 & $\begin{array}{l}\text { Nerve injury, } \\
\text { pneumothorax }\end{array}$ \\
\hline
\end{tabular}

rological symptoms or renal insufficiency. Metoclopramide acts as a D3 antagonist and 5 HT4 agonist to antiemetic effect and suppression hiccup arc, and such as anti-epileptics and gabapentin, it acts centrally to inhibit excitatory neurotransmitters in the central nervous system [13].

A randomized and placebo-controlled group trial showed that metoclopramide and baclofen were effective for intractable persistent hiccups [14]. In this case, metoclopramide $10 \mathrm{mg}$, which is known to have no serious adverse effects to other drugs, was first selected as a treatment.

Cervical epidural block, SGB, and vagus nerve block have also been reported to be effective against hiccups, but these were reported from case series.

For example, a phrenic nerve block may be performed, which may lead to a serious decrease in lung capacity and respiration volume due to nerve damage as well as epidural block and unilateral paralysis of the diaphragm [15]. It is necessary to perform under ultrasound guidance and close observation after the procedure. In the case of a persistent hiccup, treatment according to the cause is required, and in the case of a dexamethasone-induced hiccup, change to another steroid drug, and in the case of a persistent hiccup after use by combining the above drugs, SGB or continuous epidural block may be helpful. Furthermore, guidelines on the appropriate treatment strategies against hiccups are warranted through further research.

We describe a case of persistent hiccups as a complication of 
cervical selective nerve root block. The hiccups were believed to be caused by the administration of dexamethasone and were successfully treated with diazepam and metoclopramide. Depending on the cause of hiccups, medication or therapeutic intervention may be effective; however, further studies are needed.

\section{REFERENCES}

1. Marieb EN. The respiratory system. In: Marieb EN, editor. Human anatomy \& physiology. 5th ed. San Francisco (CA): Benjamin Cumming; 2001. p. 858.

2. Kanniah SK. Acute transient hiccups after epidural injection of levobupivacaine. Int J Obstet Anesth 2009;18:193-4.

3. Slipman CW, Shin CH, Patel RK, Braverman DL, Lenrow DA, Ellen MI, et al. Persistent hiccup associated with thoracic epidural injection. Am J Phys Med Rehabil 2001;80:618-21.

4. Thompson DF, Landry JP. Drug-induced hiccups. Ann Pharmacother 1997;31:367-9.

5. Dickerman RD, Jaikumar S. The hiccup reflex arc and persistent hiccups with high-dose anabolic steroids: is the brainstem the steroid-responsive locus? Clin Neuropharmacol 2001;24:62-4.

6. Abubaker AK, Rabadi DK, Kassab M, Al-Qudah MA. Persistent hiccups after cervical epidural steroid injection. Am J Case Rep 2018;19:397-9.

7. Iijima M, Uchigata $M$, Ohashi $T$, Kato $H$. Intractable hiccups induced by high-dose intravenous methylprednisolone in a patient with multiple sclerosis. Eur J Neurol 2006;13:201-2.

8. Odonkor CA, Smith B, Rivera K, Chhatre A. Persistent singultus associated with lumbar epidural steroid injections in a septuagenarian: a case report and review. Am J Phys Med Rehabil 2017;96:e1-4.

9. El-Boghdadly K, Chin KJ, Chan VWS. Phrenic nerve palsy and regional anesthesia for shoulder surgery: anatomical, physiologic, and clinical considerations. Anesthesiology 2017;127:173-91.

10. Lee AR, Cho YW, Lee JM, Shin YJ, Han IS, Lee HK. Treatment of persistent postoperative hiccups with stellate ganglion block: three case reports. Medicine (Baltimore) 2018;97:e13370.

11. Steger M, Schneemann M, Fox M. Systemic review: the pathogenesis and pharmacological treatment of hiccups. Aliment Pharmacol Ther 2015;42: 1037-50.

12. Lee JH, Kim TY, Lee HW, Choi YS, Moon SY, Cheong YK. Treatment of intractable hiccups with an oral agent monotherapy of baclofen: a case report. Korean J Pain 2010;23:42-5.

13. Porzio G, Aielli F, Verna L, Aloisi P, Galletti B, Ficorella C. Gabapentin in the treatment of hiccups in patients with advanced cancer: a 5-year experience. Clin Neuropharmacol 2010;33:179-80.

14. Wang T, Wang D. Metoclopramide for patients with intractable hiccups: a multicentre, randomised, controlled pilot study. Intern Med J 2014;44: 1205-9.

15. Kang SS, Jang JS, Park JH, Hong SJ, Shin KM, Yun YJ. Unilateral phrenic nerve block guided by ultrasonography and nerve stimulator for the treatment of hiccup developed after tongue cancer operation: a case report. Korean J Anesthesiol 2009;56:208-10. 